\title{
Filamentation and Forward Brillouin Scatter of Entire Smoothed and Aberrated Laser Beams
}

C.H. Still, R.L. Berger, A.B. Langdon, D.E. Hinkel, L.J. Suter, and E.A. Williams

This article was submitted to the $41^{\text {st }}$ Annual Meeting of the Division of Plasma Physics, Seattle, WA, November 15-19, 1999

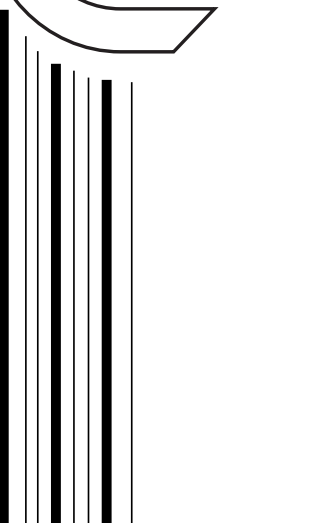




\section{DISCLAIMER}

This document was prepared as an account of work sponsored by an agency of the United States Government. Neither the United States Government nor the University of California nor any of their employees, makes any warranty, express or implied, or assumes any legal liability or responsibility for the accuracy, completeness, or usefulness of any information, apparatus, product, or process disclosed, or represents that its use would not infringe privately owned rights. Reference herein to any specific commercial product, process, or service by trade name, trademark, manufacturer, or otherwise, does not necessarily constitute or imply its endorsement, recommendation, or favoring by the United States Government or the University of California. The views and opinions of authors expressed herein do not necessarily state or reflect those of the United States Government or the University of California, and shall not be used for advertising or product endorsement purposes.

This is a preprint of a paper intended for publication in a journal or proceedings. Since changes may be made before publication, this preprint is made available with the understanding that it will not be cited or reproduced without the permission of the author.

This report has been reproduced directly from the best available copy.

Available electronically at http://www.doc.gov/bridge

Available for a processing fee to U.S. Department of Energy

And its contractors in paper from

U.S. Department of Energy

Office of Scientific and Technical Information

P.O. Box 62

Oak Ridge, TN 37831-0062

Telephone: (865) 576-8401

Facsimile: (865) 576-5728

E-mail: reports@adonis.osti.gov

Available for the sale to the public from

U.S. Department of Commerce

National Technical Information Service

5285 Port Royal Road

Springfield, VA 22161

Telephone: (800) 553-6847

Facsimile: (703) 605-6900

E-mail: orders@ntis.fedworld.gov

Online ordering: http://www.ntis.gov/ordering.htm

OR

Lawrence Livermore National Laboratory

Technical Information Department's Digital Library

http://www.llnl.gov/tid/Library.html 


\title{
Filamentation and forward Brillouin scatter of entire smoothed and aberrated laser beams*
}

\author{
C. H. Still, ${ }^{\dagger}$ R. L. Berger, A. B. Langdon, D. E. Hinkel, L. J. Suter, and E. A. Williams \\ Lawrence Livermore National Laboratory \\ 7000 East Avenue, L-031 \\ Livermore, CA 94550 \\ (Draft as of Fri Oct 29 11:31:02 PD'T 1999)
}

\begin{abstract}
Laser-plasma interactions are sensitive to both the fine-scale speckle and the larger scale envelope intensity of the beam. For some time, simulations have been done on volumes taken from part of the laser beam cross-section, and the results from multiple simulations extrapolated to predict the behavior of the entire beam. However, extrapolation could very well miss effects of the larger scale structure on the fine-scale. The only definitive method is to simulate the entire beam. These very large calculations have been infeasible until recently, but they are now possible on massively parallel computers. Whole beam simulations show the dramatic difference in the propagation and break up of smoothed and aberrated beams.
\end{abstract}

\footnotetext{
*Work performed under the auspices of the U. S. DoE by LLNL under contract No. W-7405-ENG48.

†bertstill@llnl.gov
} 


\section{INTRODUCTION}

Because of the increased laser intensity and longer path lengths present in experiments on the National Ignition Facility (NIF), understanding the effects of laser-plasma interactions (LPI) will be more important in successfully achieving ignition. Both the fine-scale speckle and the larger scale envelope intensity of the beam heavily influence LPI. Previously, results from several simulations performed on volumes taken from part of the laser beam crosssection have been combined to extrapolate the behavior of a full-scale calculation. However, there is the potential for inherent inaccuracies in this approach, and important interaction effects of the large-scale structure on the fine-scale speckles are neglected. The only definitive method of including these effects is to perform simulations on the entire beam.

Since NIF is still under construction, and several years away from fielding experiments, simulations of NIF conditions are performed on the NOVA laser at Lawrence Livermore National Laboratory and the Omega laser at the Laboratory for Laser Energetics (University of Rochester). We can simulate the LPI present in these experiments numerically and then benchmark our results against the actual experiments. Numerical simulations use measurements taken from one of the NOVA beamlines and plasma conditions from experimental data or LASNEX ${ }^{17}$ calculations. To our knowledge, there are no other whole beam simulations of laser-plasma interactions (LPI) which include all these lentgh scales.

F3D is a well-documented 3D laser propagation hydrocode (see, e. g., Berger, et al. ${ }^{3}$ and Still, et al. ${ }^{15}$ ). It also includes models for stimulated Brillouin and Raman backscatter ${ }^{4}, 5$. In order to be able to simulate larger plasma volumes, we are redesigning F3D to run on massively parallel processors (MPPs). ${ }^{1}$ Using the MPP code pF3d, we have simulated whole NOVA beam filamentation, beam bending, and forward SBS. These calculations are large, requiring 1.5 billion zones for a NOVA beam sized plasma (a data set of around 120 gigabytes) and running for a few days on a large MPP.

In this article, we will discuss the beam models, then present whole beam simulation results demonstrating the dramatic difference in the propagation of smoothed and unsmoothed 
laser beams. A discussion of the massively parallel simulation code is included in the appendix for completeness.

\section{LASER BEAM MODELS}

The electric field input to the simulation code is generated using measurements taken from the final optics assembly of a beamline in the NOVA laser. Fig. 1 shows a schematic of the setup. The laser beam passes through the final focusing lens and then through a phase plate for smoothed beams. The beam measurements are used to generate a near-field input to the pF3d simulation code. pF3d takes the input electric field and the initial plasma conditions and self-consistently simulates the evolution of the beam propagating through the plasma. The electric field on the output plane can be used to simulate experimental beam diagnostics such as the transmitted frequency spectra and near-field image.

Fig. 2 shows fringes of the laser field for an unsmoothed $f / 8$ beam and two types of spatially smoothed beams. The images exhibil demarcation lines where the nine KDP (potassium dihydrogen phosphate) crystals abut, a horizontal line where the NOVA amplifier disk is split, and a central occlusion which serves to block unconverted laser light from illuminating the target. The image of the unsmoothed beam clearly shows the aberrations present in a NOVA beam. ${ }^{16}$ The other image in Fig. 2 is a composition of imagcs from two types of smoothed beams.

A random phase plate (RPP) is an array of elements with phases of either 0 or $\pi$ randomly distributed over the plate. An RPP beam produces a series of rings so that the central spot has an envelope approximated by a sinc ${ }^{2}$ function. The upper half of the smoothed beam image is taken from an RPP beam, where the NOVA aberrations have been superimposed on an RPP.

The other type of beam smoothing uses a kinoform phase plate (KPP). Becanse of the sinc $^{2}$ envelope, an RPP beam carries $83 \%$ of the total beam power in the central spot, and the spot is centrally peaked. The KPP is designed to correct these problems, carrying 
greater than $90 \%$ of the beam power in a spot with a flat-topped envelope. The lower half of the smoothed beam image in Fig. 2 shows the NOVA aberrations with an $f / 8 \mathrm{KPP}$ beam. ${ }^{7}$

F3D shows how propagation through plasma modifies the beam structure. The three images in Fig. 3 show the intensity in transverse $k$-space, $|E(k)|^{2}$, resulting from an $f / 8$ KPP bcam passing through a plasma slab of varying thickness. This corresponds to the image at the transmitted light relay plane. The plasma used is the canonical slab for NIFignition conditions, $0.1 n_{c} \mathrm{C}_{5} \mathrm{H}_{12}$ with electron temperature $3 \mathrm{keV}$, and the beam used has a wavelength of $351 \mathrm{~nm}$ and an intensity $2 \times 10^{15} \mathrm{~W} / \mathrm{cm}^{2}$. The first image corresponds to vacuum propagation without plasma. The beam still retains most of its structure with a fairly thin plasma, as seen in the second image (with $105 \mu \mathrm{m}$ of plasma), but the KPP near field structure is mostly lost at the end of a $1.1 \mathrm{~mm}$ plasma, as seen in the third image. We now wish to compare whole beam simulations of the three beam types (unsmoothed, KPP, and RPP).

\section{WHOLE BEAM SIMULATIONS}

Experiments on NOVA and OMEGA are designed to simulate one or more beams in a NIF hohlraum experiment. Using the beam models, discussed in Section II, we can numerically simulate the entire beam. By taking a $1 \mathrm{~mm}$ slab plasma of neopentane $\left(\mathrm{C}_{5} \mathrm{H}_{12}\right)$ at $0.1 n_{c}$, with initial electron temperature $3 \mathrm{keV}$ and ion temperature $1 \mathrm{keV}$, we can simulate the propagation of three different beam types with NOVA aberrations, an unsmoothed beam, one with KPP smoothing, and one with RPP smoothing.

Fig. 4 shows the beam intensity at the incident plane of the simulated plasma for

each of the three beams. In every case, an equivalent spot size was chosen for a beam power of $2 \mathrm{TW}$ in order to achieve a spot-averaged intensity of $2 \times 10^{15} \mathrm{~W} / \mathrm{cm}^{2}$. For the unsmoothed beam, the spot size is achieved at $2 \mathrm{~mm}$ beyond best focus. The KPP (Fig. 4a) has fine-scale structure with many small hotspots distributed throughout, and has a squarish envelope. By comparison, the RPP spot is more rounded than the KPP spot (the 
RPP envelope is approximated by a raised cosine function to make the intensity drop to zero smoothly at the edge before adding the beam aberrations), and also has many small speckles distributed throughout, but there are some hotspots more intense than any in the KPP. These most intense hotspots are centrally located in the RPP spot(Fig. 4b). The presence of the NOVA aberrations produces the vertical fuzz at the edges of the KPP and RPP spots. The unsmoothed beam has a completely different envelope, only vaguely round towards the center (Fig. 4c). There are fewer speckles, each of which carries more energy than the hotspots of the KPP or the RPP. The spatial profiles of smoothed intensity for the three beams show these qualities (Fig. 5): the KPP has a flat-topped distribution; the RPP is centrally peaked; and the unsmoothed beam exhibits an unorganized structure.

Differences in beam propagation are apparent, as shown in Fig. 6 at 32 ps. Within the first quarter of the simulation volume, the KPP beam exhibits filamentation, with its hotter speckles breaking up. Most of the beam retains its original character, so there is only a little spreading (Fig. 6a). There is comparatively more spreading in the RPP beam, as shown in Fig. 6b. The RPP spot is slightly narrower than the KPP spot at the entrance plane (the full-width-half-maximum ${ }^{9}$ of the RPP is $216 \mu \mathrm{m}$ versus $377 \mu \mathrm{m}$ for the KPP), but the transmitted spots have similar width (FWHM of the RPP is $368 \mu \mathrm{m}$ versus $424 \mu \mathrm{m}$ for the KPP). The central hotspots of the RPP carry more energy than hotspots in the KPP, and therefore, break into smaller filaments. By contrast to the smoothed beams, the unsmoothed beam breaks up completely (Fig. 6c). The very large hotspots in the unsmoothed beam carry much more power than hotspots in either smoothed beam, causing them to break into very small filaments within the first 300-500 wavelengths of the simulation box. Note the fine-scale structure present at a distance of 500-1000 wavelengths from the entrance plane in Fig. 6c.

Transverse slices taken at the output plane for the KPP and RPP beams (Fig. 7a and Fig. 7b, respectively) and at the midpoint of the simulation for the unsmoothed beam (Fig. 7c) complete the story. The spreading in $y$-direction of the smoothed beams is due to the NOVA aberrations, and the $x$-direction spreading has been largely suppressed by the beam 
smoothing. At half the propagation distance, the unsmoothed beam has spread to fill the simulation volume. Fig. 8 shows the smoothed spatial profiles for the three beams at the output plane. Note that the spatial profile of the RPP is no longer markedly different from the KPP.

With these simulations in hand, we wish to quantify bcam brcakup. Johnston proposed using the Hamiltonian per unit beam power, assuming a stcady statc response in a slab plasma. ${ }^{10}$ Although not technically applicable in a non-steady state, we wish to sce how well this measure quantifies beam breakup in our simulations.

Assuming pressure balance and a constant density profile, the Hamiltonian for the paraxial wave equation is

$$
H(z)=\frac{1}{2 \pi} \int d x d y\left\{|\nabla u|^{2}-\frac{\omega_{p}^{2}}{\omega_{0}^{2}}\left[|u|^{2}-1+\exp \left(-|u|^{2}\right)\right]\right\}
$$

and the normalized beam power is given by

$$
N(z)=\frac{1}{2 \pi} \int|u|^{2} d x d y
$$

where

$$
u^{2}=\frac{1}{4}\left|\frac{e \hat{E}}{m_{e} \omega_{0}}\right|^{2} \frac{1}{v_{e}^{2}}
$$

is the dimensionless field if the laser field is given by

$$
E=\frac{1}{2} \hat{E}\left(e^{-i \omega_{0} t+i k_{0} z}+e^{i \omega_{0} t-i k_{0} z}\right)
$$

and

$$
\frac{\omega_{p}^{2}}{\omega_{0}^{2}}=\frac{\left\langle n_{e}(x, y, z)\right\rangle_{x, y}}{n_{c}}
$$

is the electron density averaged over an $x y$-plane relative to the critical density. If we denote the first term by

$$
H_{1}(z)=\frac{1}{2 \pi} \int|\nabla u|^{2} d x d y
$$


and the second term by

$$
H_{2}(z)=-\frac{1}{2 \pi} \int \frac{\omega_{p}^{2}}{\omega_{0}^{2}}\left[|u|^{2}-1+\exp \left(-|u|^{2}\right)\right] d x d y
$$

then $H=H_{1}+H_{2}$ and $H / N$ is a measure of beam breakup. To see this, consider the case of a single speckle. At the onset of filamentation, $H$ becomes negative as the self-focusing part $\left(H_{2}\right)$ begins to dominate the vacuum propagation part $\left(H_{1}\right)$. The more unstable to filamentation the speckle is, the larger in magnitude that $H_{2} / N$ becomes. Similarly, the more fine-scale transverse stucture the beam develops due to break up, the larger $H_{1} / N$ is. A stable speckle without much transverse structure will give rise to a positive, small or moderate value for $H / N$.

Fig. 9a and Fig. 9b show 2D $x z$-slices through two different single 3D Gaussian beams propagating through a $0.1 n_{c} \mathrm{CH}$ plasma slab with $T_{e}=1 \mathrm{keV}$ and $T_{i}=0.5 \mathrm{keV}$. Such a Gaussian could be taken to represent an isolated speckle. Fig. 9a shows a beam with peak intensity $10^{14} \mathrm{~W} / \mathrm{cm}^{2}$ at the incident plane and Fig. 9b depicts a beam 100 times more intense (peak intensity $I=10^{16} \mathrm{~W} / \mathrm{cm}^{2}$ at the incident plane). While the more intense beam is filamenting quite strongly, the less intense beam is very stable. This behavior is reflected in the diagnostic plots, Fig. 10, Fig. 11, and Fig. 12. For the less intense beam (the dotted lines) $\sqrt{N / H_{1}}$ is flat indicating not much transverse structure has developed, $\mathrm{H}_{2} / \mathrm{N}$ is negligible since the beam is stable for filamentation, and thus the resulting quantity $H / N$ is a small positive number (around $10^{-3}$ ). Conversely, the more intense beam (solid lines) breaks up as reflected by the significant drop in $\sqrt{N / H_{1}} \cdot H_{2} / N$, and consequently $H / N$, are both negative.

In a beam with many speckles, the aggregate quantities $H_{1} / N, H_{2} / N$, and $H / N$ get a contribution from each individual speckle, thus many speckles breaking into filaments implies larger magnitude values for $H_{1} / N$ and $-H_{2} / N$, and consequently $H / N$. Hence, $\sqrt{N / H_{1}}$ will be small.

For the three whole beam simulations, the results of these diagnostics follow our expectations. The transverse correlation length $\sqrt{N / H_{1}}$ drops fairly sharply in all three simulations 
indicating that beam brcakup is occurring in the front part of the plasma (Fig. 13). The unsmoothed beam breaks up the most, and the KPP beam the least. Fig. 14 shows that the unsmoothed beam has more speckles undergoing stronger self-focusing than either of the smoothed beams, and the KPP has the fewest. Finally, in Fig. 15 we see that very early in the box, the KPP is showing the most fine-scale structure, but that as the hotspots in the RPP beam and those in the unsmoothed beam break up, the other two beams develop much finer-scale transverse structure than the KPP, as indicated by a larger value in the normalized Hamiltonian.

\section{CONCLUSIONS}

The higher intensities and longer scale lengths present in the NIF laser will accentuate laser-plasma instabilities (LPI). Therefore, successful ignition is predicated on the ability to model and understand these instabilities. In order to improve the modeling capability for LPI under NIF conditions, it is necessary to simulate entire beams. While computationally difficult, whole beam simulations can be done on massively parallel computers. In this paper, we have presented the results of simulating the propagation of three different $f / 8$ NOVA beams in a $1.1 \mathrm{~mm}$ slab plasma $\left(0.1 n_{c} \mathrm{C}_{5} \mathrm{H}_{12}\right.$ at $\left.T_{e}=3 \mathrm{keV}, T_{i}=1 \mathrm{keV}\right)$ : a KPP beam, an RPP beam, and an unsmoothed beam. The unsmoothed beam propagates dramatically differently from either smoothed beam, and the two smoothed beams are different in more subtle ways.

\section{APPENDIX A: THE MASSIVELY PARALLEL SIMULATION CODE PF3D}

The development of a fully-nonlinear, time-dependent hydrodynamic and heat transport code F3D has allowed us to pursue a number of very interesting problems in laser beam self-focusing, flamentation, and laser-plasma instabilities for which the plasma density and flow velocities are strongly perturbed. 
Currently, the MPP code pF3d has paraxial light propagation, nonlinear Eulerian hydrodynamics, linearized electron heat conduction, and sophisticated laser beam models. Models for stimulated Raman and stimulated Brillouin backscatter (SRS and SBS, respectively) based on those for $\mathrm{F}_{3} \mathrm{D}^{5}$ are currently being added to the MPP code.

Because in filamentation the frequency $\omega$ and the wavenumber $k$ of the incident light are only slightly perturbed, an envelope approximation in time and space is reasonable. The resulting paraxial wave equation is

$$
\left(\frac{\partial}{\partial t}+v_{g} \frac{\partial}{\partial z}-\frac{i c^{2}}{2 \omega_{0}} \mathcal{D}_{\perp}^{2}+\frac{1}{2} \frac{d v_{g}}{d z}+\nu\right) E=-\frac{4 \pi e^{2} i}{2 \omega_{0} m_{e}} \delta n_{e} E
$$

for the complex light-wave envelope amplitude, $E$, oscillating at frequency $\omega_{0}$ and wavevector,

$$
c^{2} k_{0}^{2}=-\frac{4 \pi e^{2}}{m_{e}} \bar{n}_{e}+\omega_{0}^{2}
$$

Here, we define

$$
\begin{aligned}
\bar{n}_{e}(z) & -\left\langle n_{e}(x, y, z)\right\rangle_{x y} \\
\delta n_{e} & =n_{e}(x, y, z)-\bar{n}_{e}(z)
\end{aligned}
$$

the light wave group velocity,

$$
v_{g}(z)=\frac{c^{2} k_{0}(z)}{\omega_{0}}
$$

the inverse Bremssthrahlung absorption rate, $\nu$, and the generalized diffraction operator ${ }^{8}$,

$$
\mathcal{D}_{\perp}^{2}=\frac{2 k_{0} \nabla_{\perp}^{2}}{k_{0}+\sqrt{k_{0}^{2}+\nabla_{\perp}^{2}}}
$$

which extends validity of the paraxial equation to higher order in $k_{\perp}$. Solution of the paraxial equation $\mathrm{A} 1$ is achieved by operator splitting, as detailed in Berger et al. ${ }^{3}$.

The Eulerian hydrodynamics equations are as follows. The continuity equation is

$$
\frac{\partial}{\partial t} n_{i}+\nabla \cdot\left(\mathbf{v} n_{i}\right)=0
$$


where $n_{i}$ is the ion density, and $\mathbf{v}$ is the fluid velocity. Note that we assume quasi-neutrality of the plasma $n_{e}=Z n_{i}$. The momentum equation is

$$
\frac{\partial}{\partial t} S_{j}+\nabla \cdot\left(\mathbf{v} S_{j}\right)+\nabla \cdot \mathbf{Q}_{j}+\frac{\partial}{\partial x_{j}} P+\frac{\partial}{\partial x_{j}} P_{e}+n_{i} \frac{\partial}{\partial x_{j}} \phi=0
$$

where $\mathbf{S}=m_{i} n_{i} \mathbf{v}$ is the momentum density, $P$ is the ion pressure, $P_{e}$ is the electron pressure, $\phi$ is the ponderomotive force due to the light, and $\mathrm{Q}$ is an artificial viscosity. The ion energy equation is written in a form where ion pressure is the fundamental quantity [using the ideal gas equation of state, $\left.\frac{3}{2} P=\frac{3}{2} n_{i} T=m_{i} n_{i} \varepsilon\right]$

$$
\frac{3}{2}\left(\frac{\partial}{\partial t} P+\nabla \cdot(\mathbf{v} P)\right)+P \nabla \cdot \mathbf{v}+\mathbf{Q}: \nabla \mathbf{v}=0 .
$$

Finally, the electron energy equation is written in the linearized form

$$
n_{e}\left(\frac{3}{2} \frac{\partial}{\partial t} \delta T_{e}+T_{e} \nabla \cdot \mathbf{v}\right)=-\nabla \cdot \delta \mathbf{q}_{e}+\delta H_{e}
$$

where $\delta \mathbf{q}_{e}$ represents (nonlocal) thermal conduction and $H_{c}$ is a heat source from inverse Bremsstrahlung absorption.

The parallel method is based on domain decomposition of the simulation box in the directions transverse to laser propagation, with each separate processor performing the same calculations on different data. Communication of off-processor data is by message passing. ${ }^{12}$

Most of the runtime for a simulation is spent in the hydrodynamics package, so its efficiency is of prime importance. The hydrodynamics time advance is done by operator splitting the equations and applying finite difference solutions, except for the calculation of the nonlocal electron heat conduction which is done in $k$-space. The advection terms are handled by a second-order van Leer scheme, and the additional terms of the momentum and energy equations are handled individually, analagously to the methods in F3D (see Still, et al. $\left.{ }^{15}\right)$. With the addition of a single layer of guard cells on hydrodynamics quantities, the finite difference calculations can be carried out concurrently with minimal message traffic. In order to handle the $k$-space calculations in parallel, we have developed a parallel fast Fourier transform $\left(\mathrm{FFT}^{6}\right)$ which trades slightly more communication with large messages for improved cache performance. The electron heat conduction calculation uses this FFT. 
The numerical solution of the light propagation involves finite differences and spectral methods. Finite differences in the refraction calculation only occur in the laser propagation direction, so guard cells are not required in light quantities. The second-order spatial derivatives in A1 are treated with spectral methods, and thus also use the parallel FFT. The runtime plot scaling problem size with number of processors is flat, demonstrating very good efficiency. Because the efficiency and scalability is good, calculations can be made using large numbers of processors which enables simulations of an entire NOVA beam. 


\section{REFERENCES}

1 An MPP connects hundreds or thousands of computers, each with its own memory, by a fast communication subsystem enabling aggregate use on a single calculation.

${ }^{2}$ The density perturbation is limited in magnitude by replacing the linear response $\delta N=$ $\left(n-n_{0}\right) / n_{0}$ by $\ln (1+\delta N)$ so the total density is never negative.

${ }^{3}$ R. L. Berger, et. al., Phys. Fluids B 5, 2243 (1993)

${ }^{4}$ R. L. Berger, et. al., Phys. Fluids B X, Y (1995)

${ }^{5}$ R. L. Berger, et. al., Phys. Fluids B X, Y (1998)

${ }^{6}$ Cooley and Tkey on fast Fourier transforms.

${ }^{7}$ S. Dixit, private communication.

${ }^{8}$ M. D. Feit and J. A. Fleck, Jr., J. Opt. Soc. Am. B5, 633 (1988)

${ }^{9}$ The full-width-half-maximum (FWHM) is the width of the beam where the intensity is half of its peak value.

10 T. Johnston, Phys. Plasmas X, Y (1997).

${ }^{11}$ A. B. Langdon and B. F. Lasinski, Phys. Rev. Lett. 34, 934 (1975)

12 book by Grop, Lusk, and Skjellum.

${ }^{13}$ R. L. Berger, et. al., Phys. Fluids B 5, 2243 (1993); R. L. Berger, et. al., Phys. Rev. Lett. 75, 1078 (1995); S. Hueller, et. al., Physica Scripta; A. J. Schmitt, Phys. Fluids 31, 3079 (1988); A. J. Schmitt, Phys. Fluids B 3, 186 (1991);

${ }^{14}$ M. A. Blain, G. Bonnaud, A. Chiron, and G. Riazuelo, "Autofocalisation of filamentation d'un Faisceau Laser dans le Cadre de l'Approximation Paraxiale et Stationnaire," CEAR-5716; B. I. Cohen, et. al., Phys. Fluids B 3, 766 (1991); V. E. Zakharov, et. al., Sov. Phys. JETP 33, 77 (1971) 
${ }^{15}$ C. H. Still, et al., ICF Quarterly (1996)

${ }^{16}$ Wegner on NOVA aberrations.

${ }^{17}$ Standard G. Zimmerman reference on LASNEX. 


\section{FIGURES}

FIG. 1. Schematic of the laser beam setup. Measurements taken from the final optics assembly are used to generate a near-field input to the pF3d simulation code.

FIG. 2. Fringes for the laser field for an unsmoothed beam (left), RPP beam (right, upper half), and KPP beam (right, lower half).

FIG. 3. Intensity in transverse $k$-space resulting from from an $f / 8 \mathrm{KPP}$ beam propagating through varying plasma thicknesses, as calculated by F3D.

FIG. 4. Beam intensity at the incident plane of the simulated plasma for three different $f / 8$ NOVA beams: (a) KPP, (b) RPP, and (c) unsmoothed. Each beam carries 2 TW of power witha spot-averaged intensity of $2 \times 10^{15} \mathrm{~W} / \mathrm{cm}^{2}$.

FIG. 5. Intensity profiles for the three beams at the incident plane, unsmoothed (dotted), KPP (dashed), and RPP (solid).

FIG. 6. Beam intensity showing propagation through $1.1 \mathrm{~mm}$ of plasma for three different $f / 8$ NOVA beams: (a) KPP, (b) RPP, and (c) unsmoothed.

FIG. 7. Beam intensity at the transmission plane of the simulated plasma for three different f/8 NOVA beams: (a) KPP, (b) RPP, and (c) unsmoothed.

FIG. 8. Intensity profiles for the three beams at the transmission plane, unsmoothed (dotted), KPP (dashed), and RPP (solid).

FIG. 9. Beam intensity showing propagation of two Gaussian beams: (a) beam with incident peak intensity of $1 \times 10^{14} \mathrm{~W} / \mathrm{cm}^{2}$ shows no sign of self-focusing, and (b) beam with incident peak intensity of $1 \times 10^{16} \mathrm{~W} / \mathrm{cm}^{2}$ showing filamentation.

FIG. 10. Transverse correlation length for the beams in Fig. 9, low intcnsity (solid), high intensity (dotted). 
FIG. 11. $H_{2} / N$ for the beams in Fig. 9, low intensity (solid), high intensity (dotted).

FIG. 12. Hamiltonian per unit power $H / N$ for the beams in Fig. 9, low intensity (solid), high intensity (dotted).

FIG. 13. Transverse correlation length for the beams in Fig. 4, unsmoothed (dotted), KPP (dashed), and RPP (solid).

FIG. 14. $H_{2} / N$ for the beams in Fig. 4, unsmoothed (dotted), KPP (dashed), and RPP (solid).

FIG. 15. Hamiltonian per unit power $H / N$ for the beams in Fig. 4, unsmoothed (dotted), KPP (dashed), and RPP (solid). 


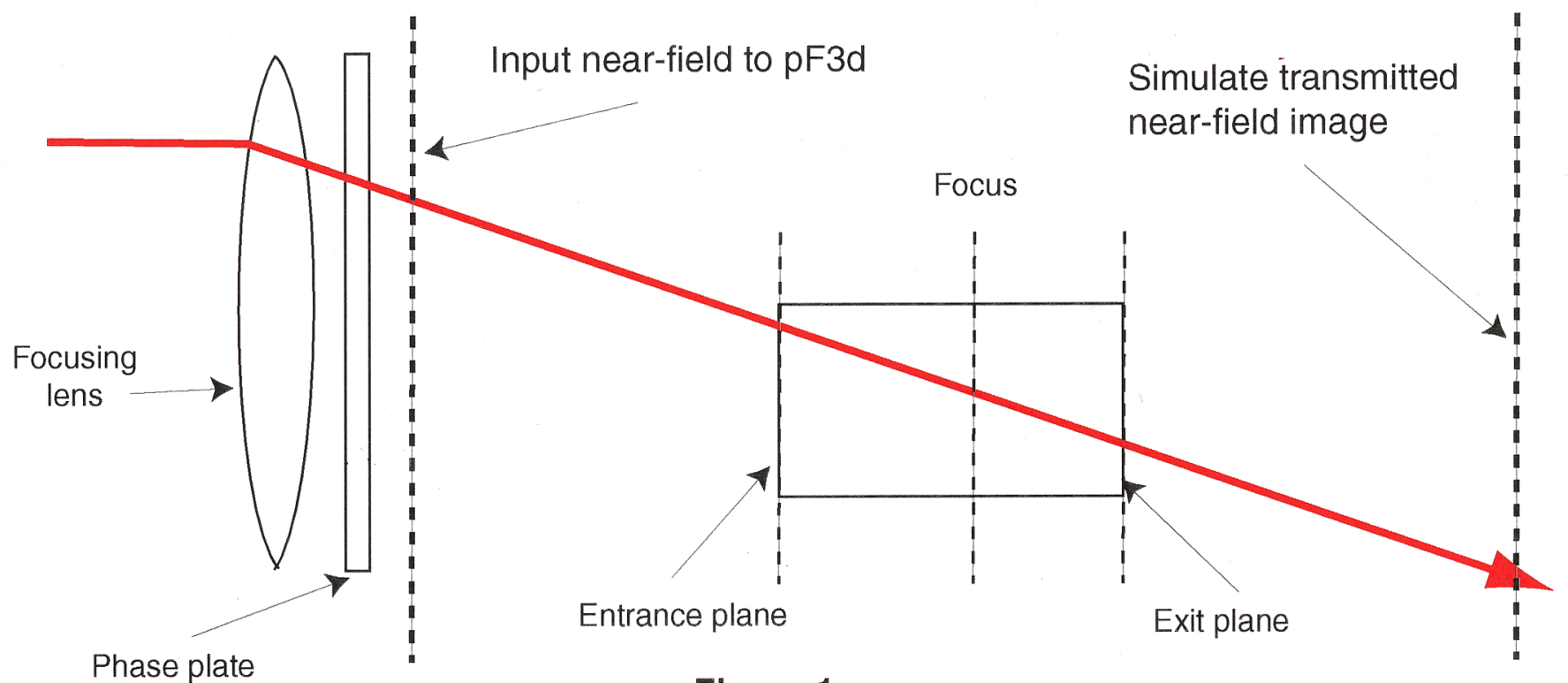

Figure 1

Unsmoothed beam

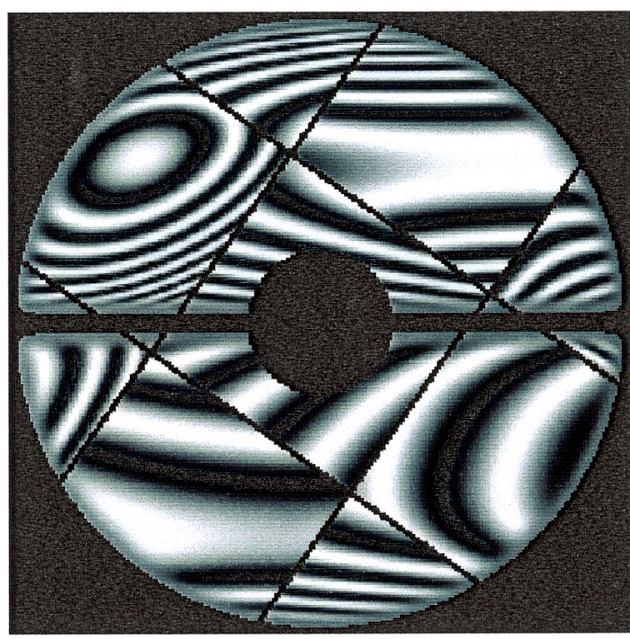

Beam with phase plates

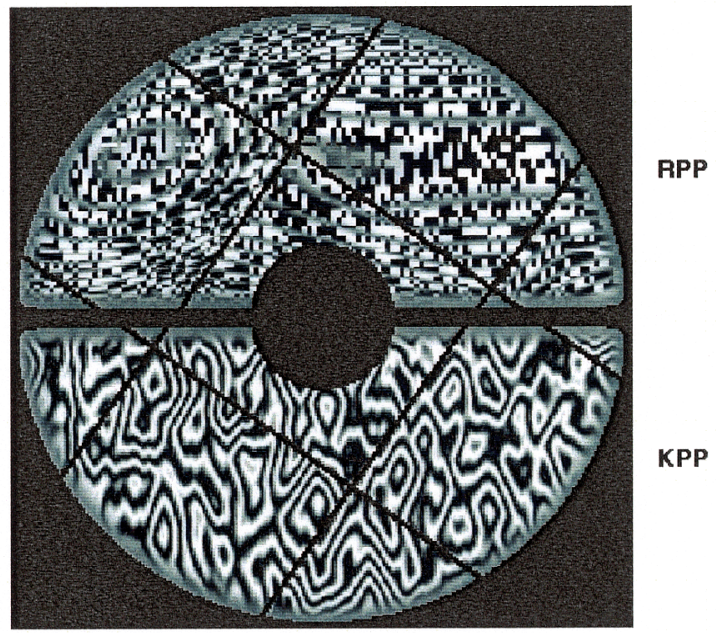

Figure 2

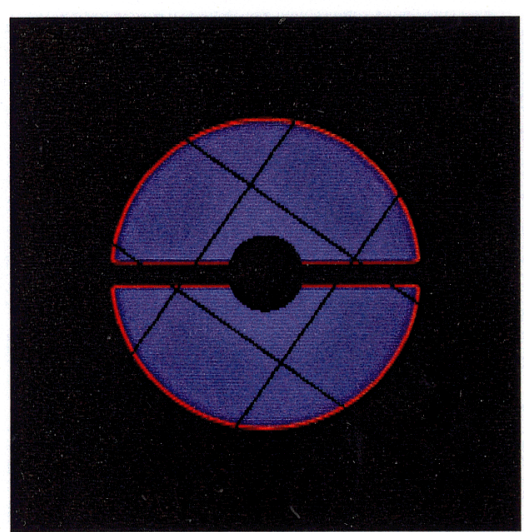

$0 \lambda$

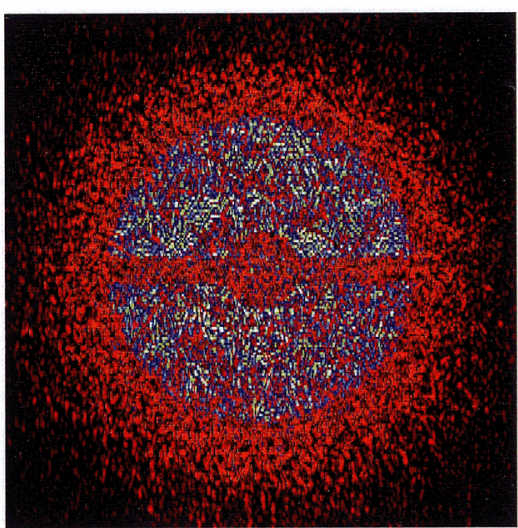

$300 \lambda$

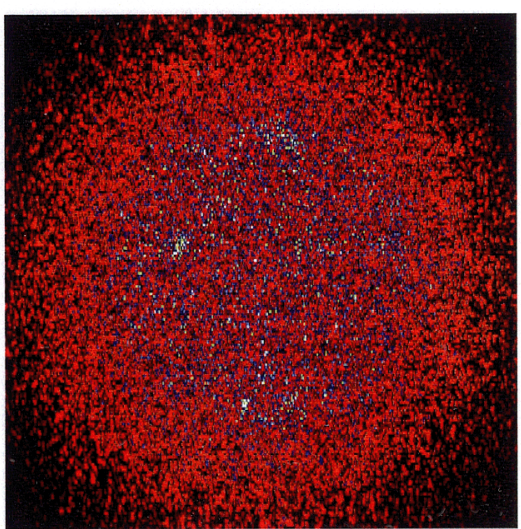

$3200 \lambda$

Figure 3 
Figure 4

a)

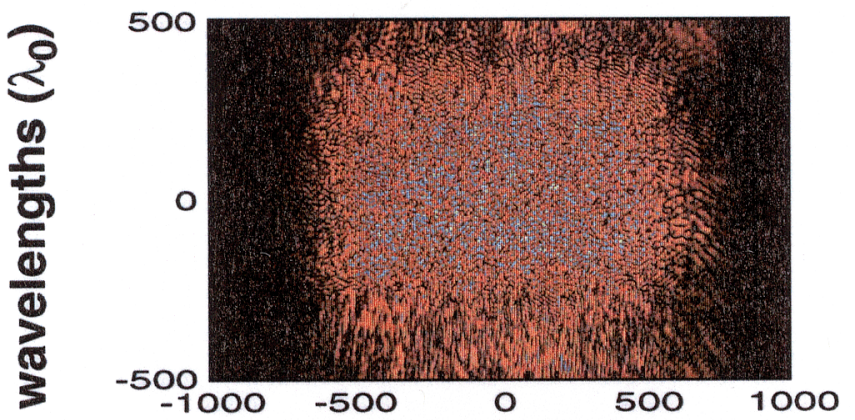

b)

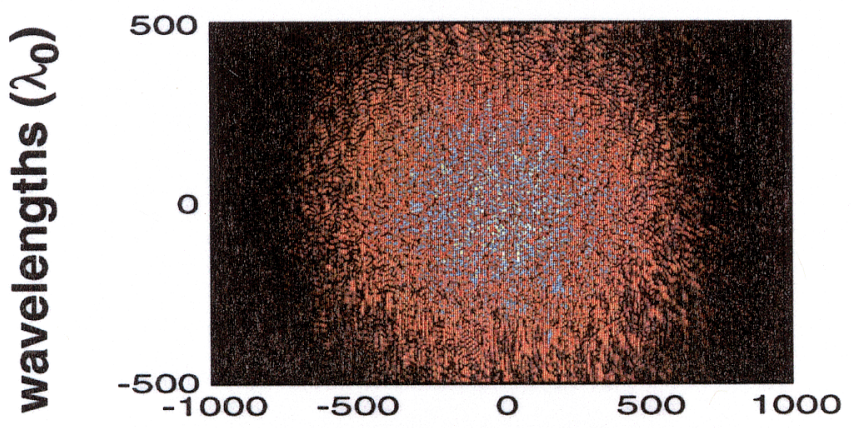

c)
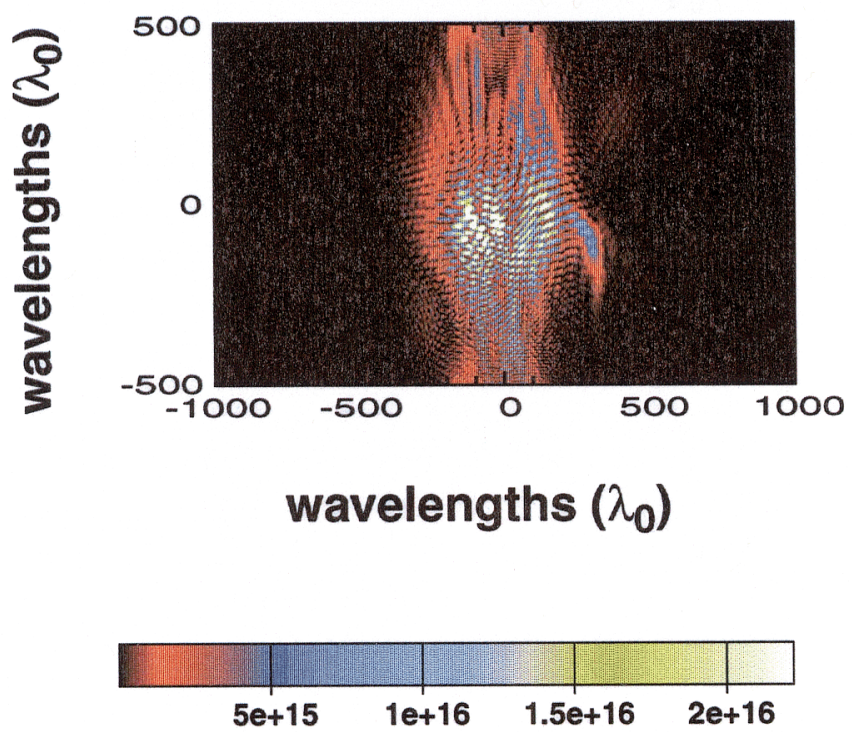
Figure 5

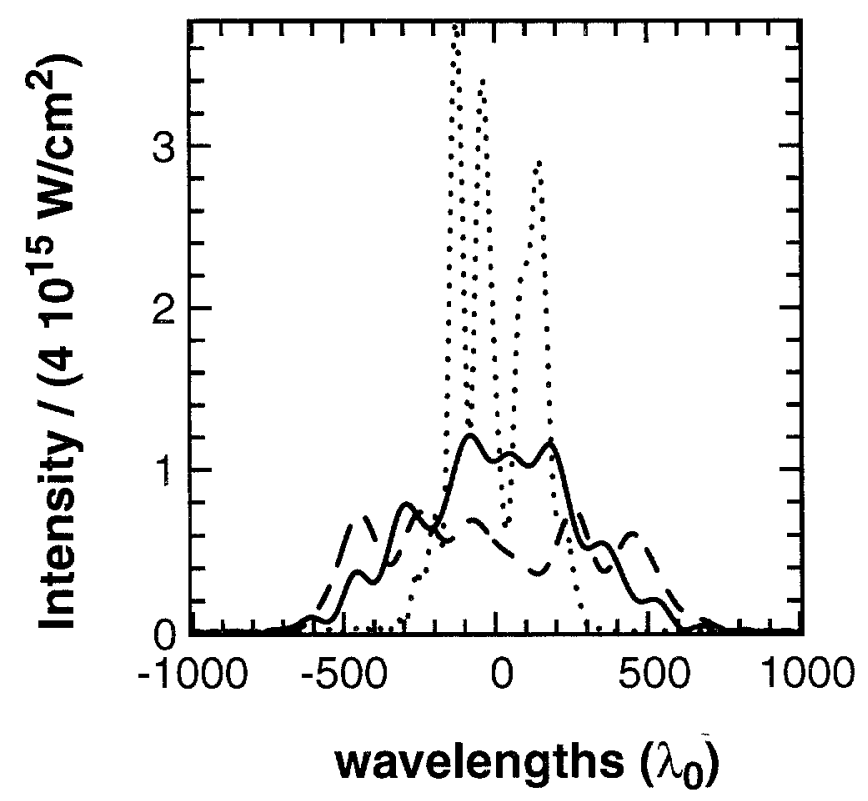


Figure 6

a)
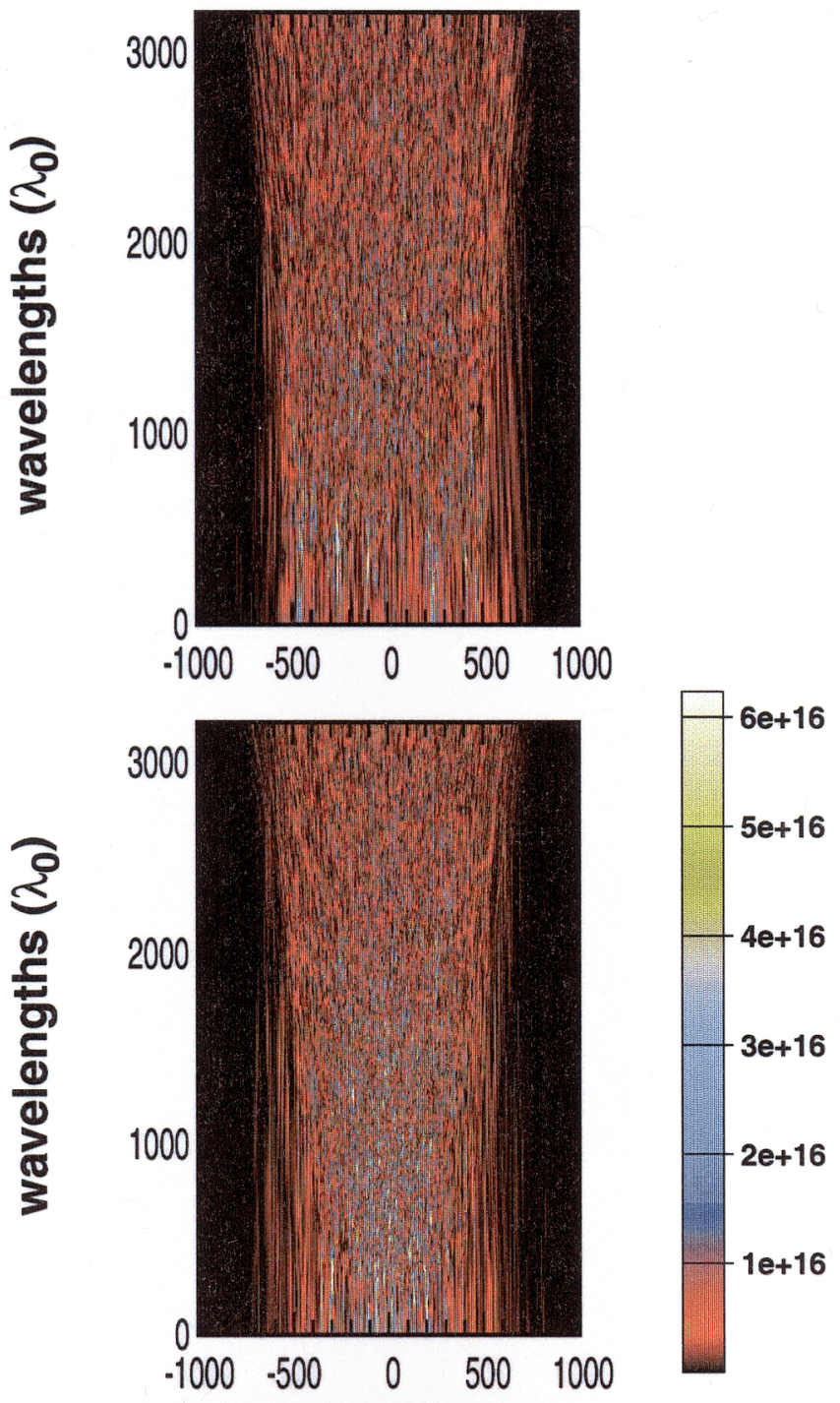

c)

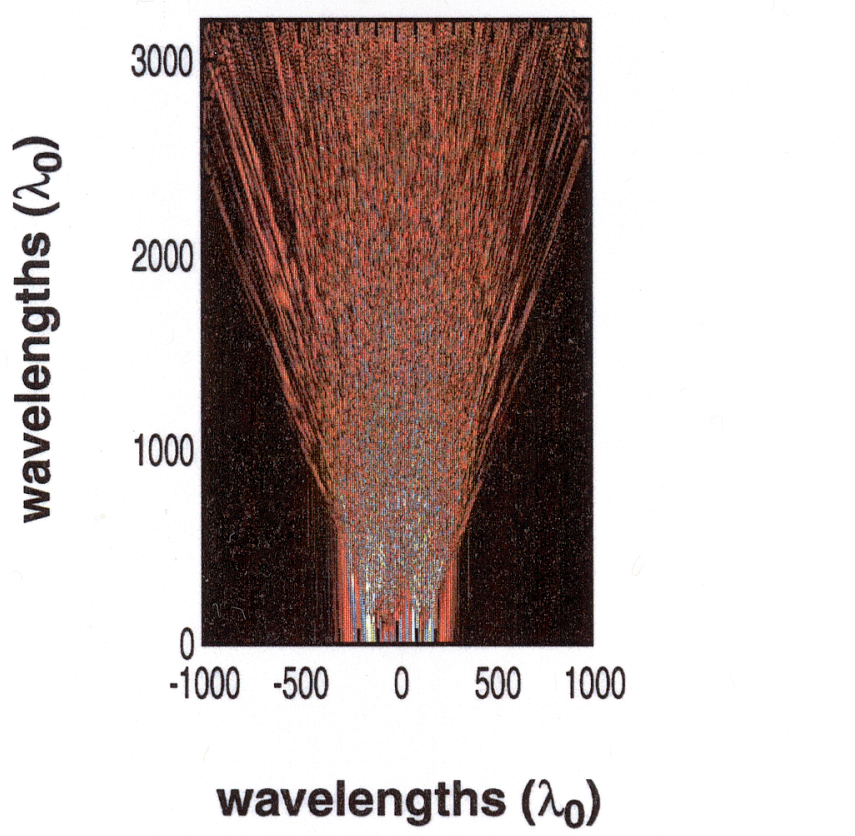


Figure 7

a)

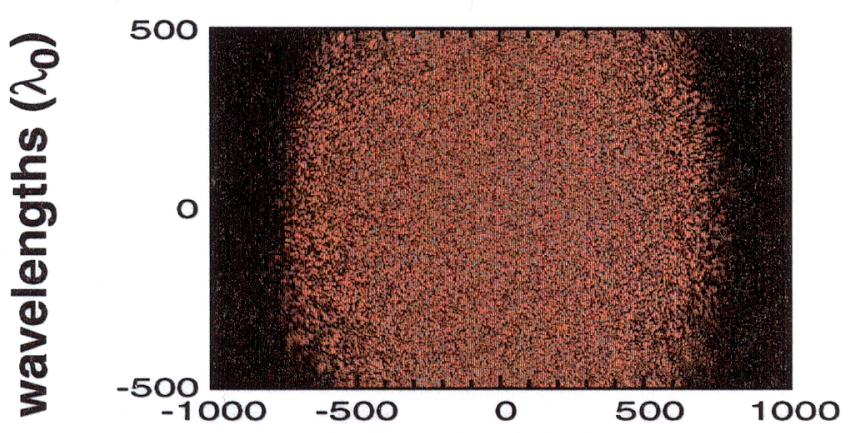

b)

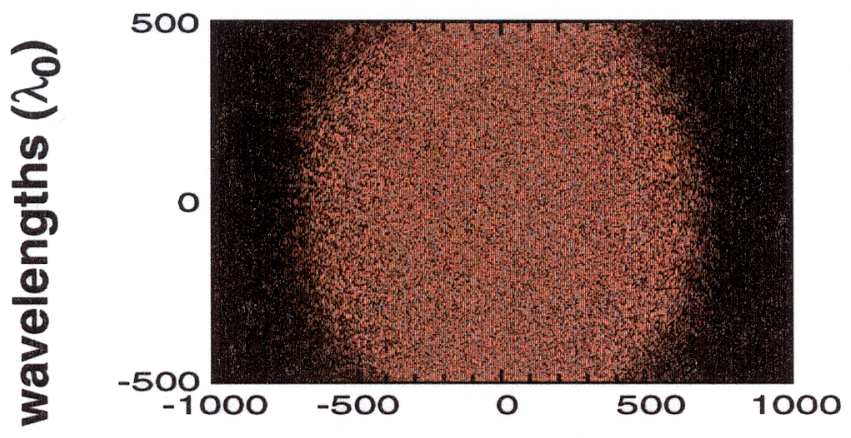

c)



wavelengths $\left(\lambda_{0}\right)$

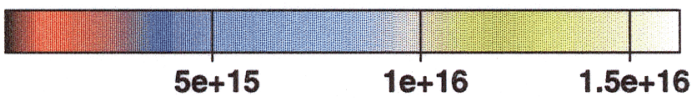


Figure 8

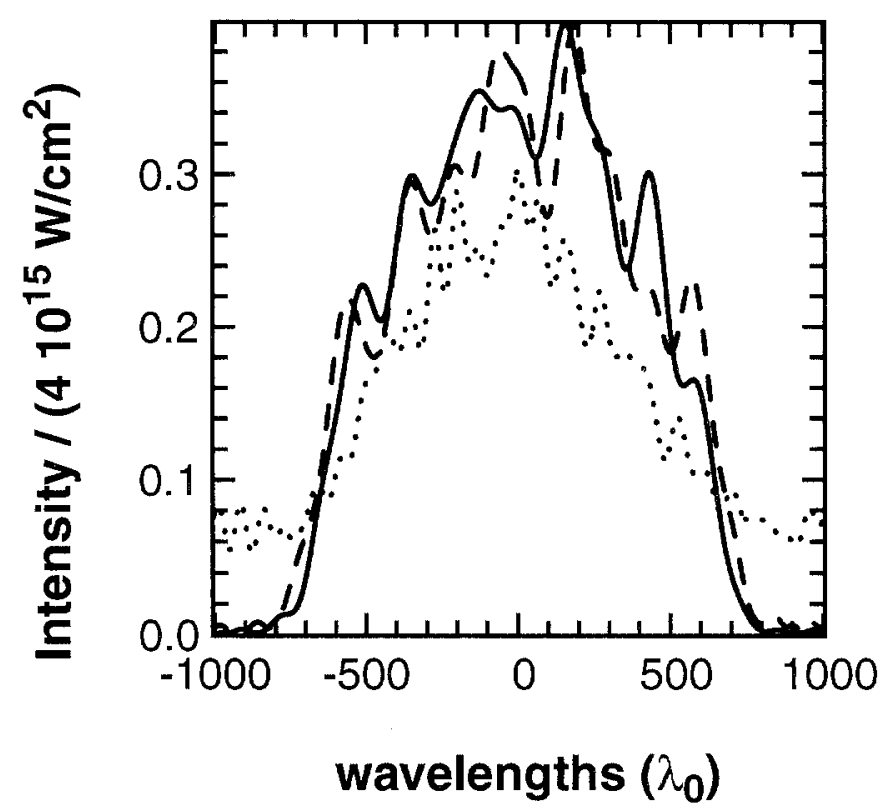


Figure 9

a)
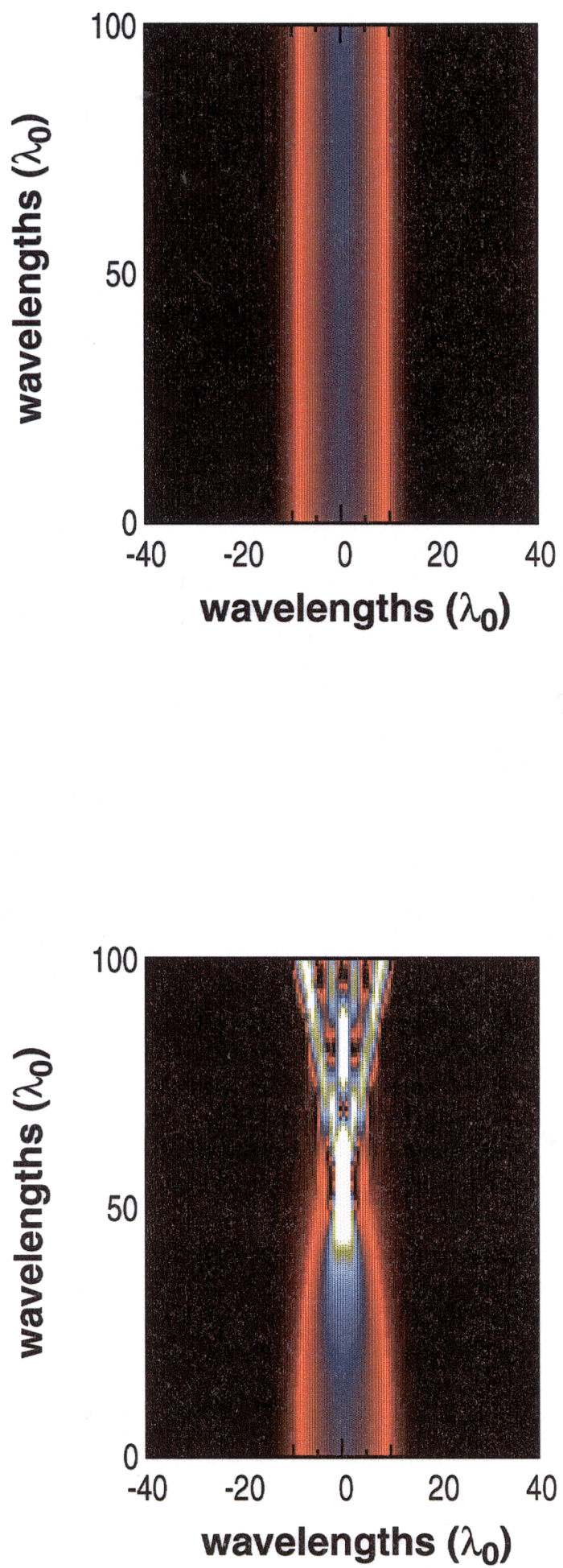
Figure 10

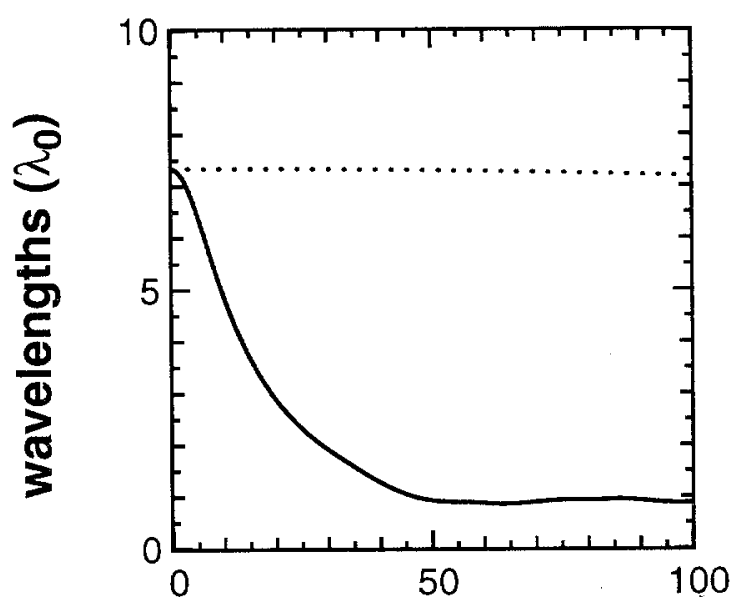

Figure 11

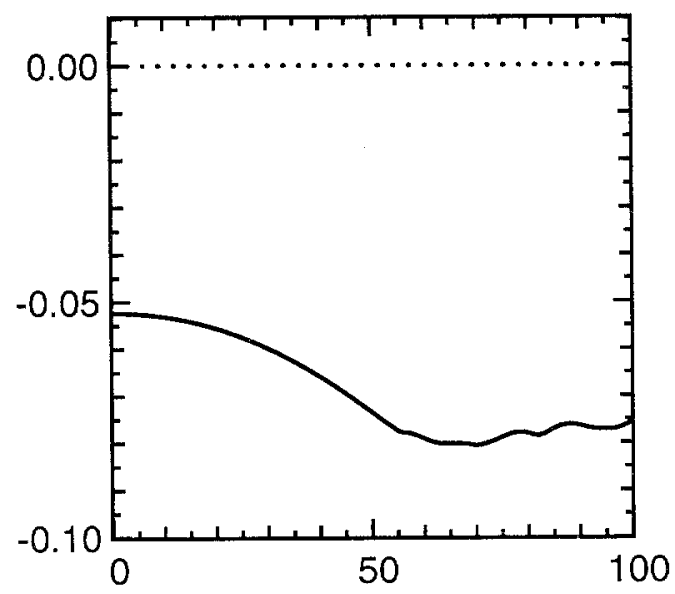

Figure 12

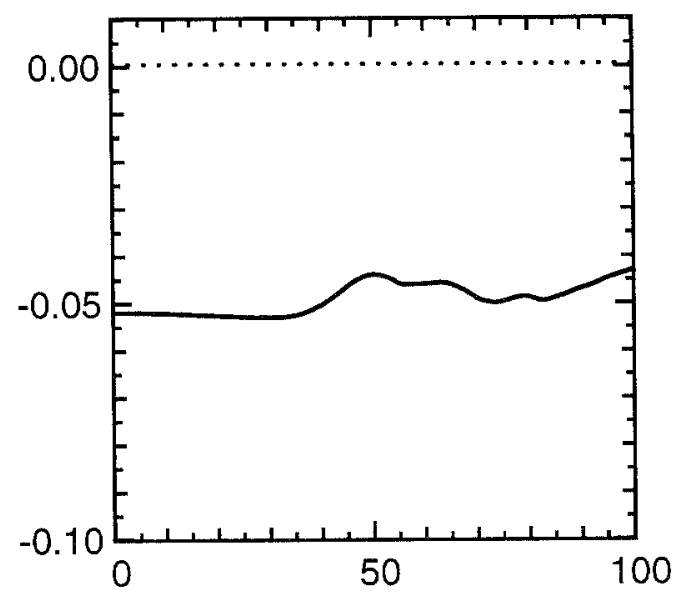

wavelengths $\left(\lambda_{0}\right)$ 
Figure 13



Figure 14

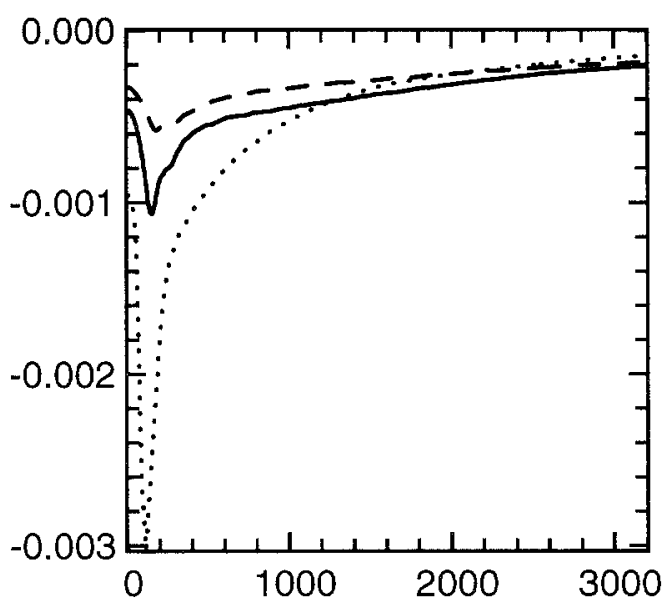

Figure 15

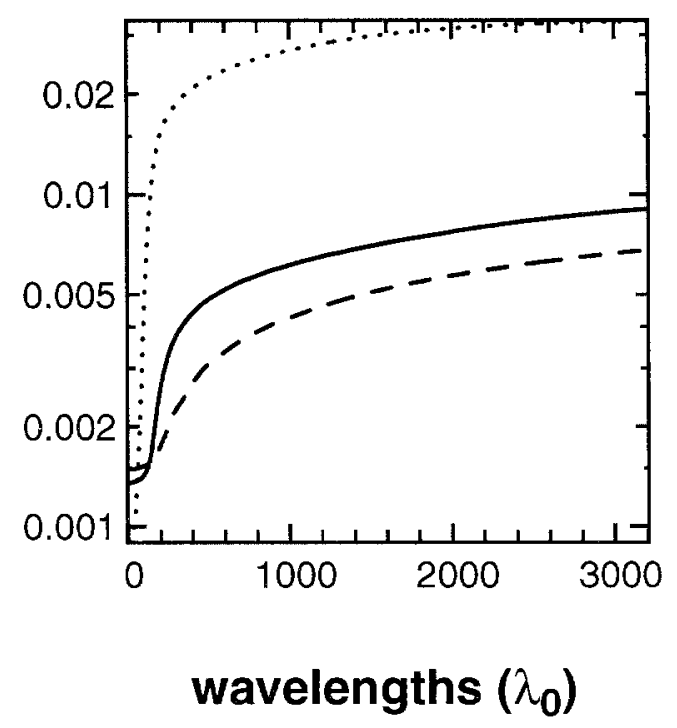

